\title{
ALTERAÇÕES DO SEPTO INTERATRIAL E ACIDENTE VASCULAR CEREBRAL ISQUÊMICO EM ADULTOS JOVENS
}

\author{
Edson M. Negrão', Ivar V. Brandi², Simone V. Nunes³, Paulo S.S. Beraldo ${ }^{4}$
}

RESUMO - Objetivo: Avaliar a freqüência de forame oval patente (FOP) em pacientes com acidente cerebrovascular isquêmico (AVCl) criptogênico ou de causa definida, investigados com doppler transcraniano (DTC) e ecocardiograma transesofágico. Paralelamente, examinar a validade diagnóstica do primeiro, comparado ao segundo método. Método: Retrospectivamente, foram estudados 124 pacientes (<51 anos), vítimas de AVCl, submetidos a DTC e ou ecocardiograma transesofágico. Os pacientes foram classificados em dois grupos: com ou sem AVCl criptogênico. Resultados: Encontramos importante associação entre AVCI criptogênico e FOP (razão de chance de 4,3; IC95\% 1,7-10,7). Foram diagnosticados apenas 5 casos de aneurisma do septo interatrial entre aqueles com FOP, todos classificados como AVCl criptogênico. Tanto a sensibilidade, especificidade e os valores preditivos positivo e negativo exibiram valores superiores a $85 \%$, semelhantes, pelo menos com base nos intervalos de confiança. Conclusão: Constatamos, pela primeira vez em nosso meio, forte associação entre AVCI criptogênico e FOP. O DTC é importante recurso diagnóstico nesse contexto, já que sua validade foi considerada muito boa, podendo ser útil no rastreamento de fontes emboligênicas, part icu l a rmente alterações do septo atrial. Uma investigação minuciosa desses casos se impõe, notadamente pela perspectiva de fechamento do shunt intracardíaco.

PALAVRAS-CHAVE: forame oval, defeitos do septo interatrial, acidente cerebrovascular, ecocardiografia transesofagiana, ultra-sonografia doppler transcraniana.

\begin{abstract}
Abnormalities of interatrial septum and ischemic stroke in young people
ABSTRACT - Objective: To verify the frequency of patent foramen ovale (PFO) among patients with ischemic stroke (cryptogenic or with a known cause) investigated by transcranial doppler (TCD) and transesophageal echocardiography. Secondarily, to determine the diagnostic validity of the former, compared with the later method. Method: Retrospectively, 124 patients ( $<51$ years old) with ischemic stroke were submitted to TCD and or transesophageal echocardiography. The patients were classified as cryptogenic stroke or not. Results: We could found an important association between cryptogenic ischemic stroke and PFO (odds ratio 4.3 - Cl 95\% 1.7 - 10.7). Only five cases of interatrial septal aneurysm were diagnosed among patients with PFO. Sensitivity, specificity and positive and negative predictive values exhibited values upper of $85 \%$, equivalents, at least based on confidence intervals. Conclusion: We could determine, for the first time in our country a strong association between cryptogenic ischemic stroke and PFO. The TCD is a valuable diagnostic reso u rce in this context since its validity was considered excellent. A detailed investigation in these cases should always be done due to the possibility of FOP closure.
\end{abstract}

KEY WORDS: patent foramen ovale, atrial heart septal defects, stroke, transesophageal echocardiography, transcranial doppler.

O acidente vascular cerebral isquêmico (AVCI) está dentre as principais causas de morbimortalidade em nosso país. Segundo dados do DATASUS, as doenças cere brovascularesre $p$ resentam a principal causa de morte no Brasil'. Esse quadro tornase mais significativo quando se instala em pacientes com menos de 60 anos, ainda economicamente ativos. Estima-se que $15 \%$ dos casos de AVCl sejam cardioembólicos ${ }^{2}$. As principais causas são a fibrilação atrial não valvar, a disfunção ventricular esquerda e a doença cardíaca reumática ${ }^{3}$. Nas duas últimas décadas, vem se destacando nesse contexto

\footnotetext{
${ }^{1}$ Médico, Cardiologista, Centro SARAH de Formação e Pesquisa, Programa de Pós-Graduação em Ciências da Reabilitação, Hospital SARAH Centro/DF; ' ${ }^{2}$ Médico, Neurologista, Hospital SARAH Salvador/BA; ${ }^{3}$ Médico, Neurologista, Hospital SARAH Belo Horizonte/MG; ${ }^{4}$ Médico, Doutor em Clínica Médica, Centro SARAH de Formação e Pesquisa, Programa de Pós-Graduação em Ciências da Reabilitação, Hospital SARAH Centro/DF.
}

Recebido 22 Abril 2005, recebido na forma final 13 Julho 2005. Aceito 31 Agosto 2005. 
a embolia paradoxal ${ }^{4}$. Alterações do septo interatrial, notadamente o forame oval patente (FOP), foram descritos como o principal exemplo desse mecanismo ${ }^{4}$. Essa condição de risco assume maior importância em jovens, uma vez que a embolia paradoxal parece ser um dos principais mecanismos para a instalação do AVCI nessa faixa etária ${ }^{4}$. Devese considerar também o risco de um segundo episódio isquêmico e a disponibilidade de tratamento para essa condição ${ }^{5}$. Quando o AVCl é considerado criptogênico a associação com FOP é ainda maior'. Atualmente, considera-se como padrão-ouro para o diagnóstico e avaliação do FOP o ecocardiogra ma transesofágico, associado ao teste de bolhas ${ }^{6}$, embora o doppler transcraniano (DTC) venha sendo cada vez mais indicado como exame inicial na detecção de shunts direito-esquerdo ${ }^{7}$.

$\mathrm{Na}$ base de dados do MEDLINE e LILACS (Literatura Latino-americana e do Caribe em Ciências da Saúde), utilizando a combinação dos descritores na língua inglesa (e seus correspondentes em português) patent foramen ovale, transesophageal echocardiography, stroke, embolism e Brazil,até junho de 2005, há registro de apenas dois artigos nacionais abordando esse tema, porém com enfoque na utilidade da ecocardiografia transesofágica na avaliação de fonte emboligênica no $\mathrm{AVCl}^{8} \mathrm{e}$ na experiência com fechamento percutâneo da comunicação interatrial e FOP, independente da presença de $\mathrm{AVCl}^{9}$.

O presente estudo tem por objetivo comparar a freqüência de alterações do septo interatrial, em pacientes com até 51 anos de idade, vítimas de $\mathrm{AVCl}$ ou ataque isquêmico transitório (AIT). Secundariamente, também buscamos determinar, no nosso meio, a utilidade do DTC na investigação de fontes emboligênicas nesses casos.

\section{MÉTODO}

Entremaio de 2003 e junho de 2004, como critério de inclusão no presente estudo, todos os pacientes consecutivos com AVCl ou AIT, diagnosticados até os 51 anos de idade, admitidos nas unidades de Brasília, São Luís, Salvador e Fortaleza, integrantes da Rede Sarah de Hospitais de Reabilitação, foram considerados. Para definição etiológica, além da avaliação clínica e neurológica, todos os pacientes seguiram o mesmo protocolo, que constou dos seguintes exames: hematológicos básicos, dosagem de homocisteína sérica, pesquisa de trombofilias e colagenoses, bem como sorologias para doença de Chagas e VDRL. Os exames de imagem incluíram, de forma isolada ou associada, conforme o caso, tomografia computadorizada, ressonância magnética e angiore s- sonância de encéfalo, doppler de artérias vertebrais e carótidas, ecocardiograma transtorácico e arteriografia ce rebral. Os casos cuja avaliação foi considerada incompleta foram excluídos da presente análise.

Especificamente, com a finalidade de investigar a p resença de shunt direito-esquerdo,todos os pacientes foram submetidos a ecocardiograma transesofágico e/ou DTC, ambos sensibilizados com o teste de bolhas. Para efeito do estudo de associação, pacientes que realizaram apenas o DTC, cujo teste de bolhas foi negativo, foram categorizados como não portadores de forame oval patente. Da mesma forma, ainda nesse contexto, foram excluídos dessa análise aqueles pacientes que apresentaram teste de bolhas positivo no DTC e não realizaram o ecocardiograma transesofágico, uma vez que não seria possível a confirmação e a definição da causa da positividade do teste de bolhas.

Para efeito do estudo de validação do DTC somente foram considerados aqueles pacientes submetidos também ao ecocardiograma transesofágico. A ordem desses exames não foi sistematizada, sendo que, na condução do segundo exame, não houve uma preocupação formal em mascarar o examinador para o resultado do primeiro. Da mesma forma, não foi restringido aos examinadores o acesso ao prontuário dos pacientes, previamente a condução dos testes diagnósticos. O Comitê de Ética da Instituição avaliou e aprovou, sem restrições, a presente investigação.

Os ecocardiogramas foram realizados por um único examinador (EMN) que, periodicamente, visitava os hospitais da rede com essa finalidade. Os equipamentos de ecocardografia utilizados foram da marca ATL, modelo HDI 5000, com sonda transesofágica multiplanar. No DTC os equipamentos utilizados foram da marca PionnerNicolet, modelo 530, e o exame foi realizado segundo técnica padrão ${ }^{10}$.

Aneurisma do septo interatrial foi definido como movimento do septo superior ou igual a $10 \mathrm{~mm}$ para qualquer um dos lados. O teste de bolhas, realizado tanto no ecocardiograma transesofágico como no DTC, foi padronizado da seguinte maneira: uma seringa com 9 $\mathrm{mL}$ de soro fisiológico era conectada a outra, por um sistema de três vias, contendo $1 \mathrm{~mL}$ de ar; o conteúdo de ambas era misturado até obter-se uma solução homogênea que era então, rapidamente, injetada através de um acesso venoso calibroso no membro superior. $O$ p rocedimento era conduzido com o paciente em repouso e associado à manobra de Valsalva. No ecocardiogra ma transesofágico o teste de bolhas foi definido como positivo quando eram visualizadas pelo menos três mic robolhas no interior do átrio esquerdo, até o quinto ciclo cardíaco após a opacificação máxima do átrio direito. No caso de até 9 microbolhas no átrio esquerdo, o shunt foi considerado pequeno; moderado, entre 10 e 29 microbolhas; e importante, acima de 30 microboIhas $^{11}$. No DTC o teste foi considerado positivo quando 
era observado pelo menos um sinal hiperintenso precoce (até 10 segundos após a infusão das microbolhas) ${ }^{7}$.

Os AVCls e os AITs foram classificados, do ponto de vista etiológico, por dois neurologistas (IVB e SVN), de forma independente, de acordo com os critérios de TOAST $^{12}$, adaptados para este estudo (Tabela 1 ). No caso de discordância nessa classificação, a decisão final foi por consenso. Para efeito de análise, AVCI associado a trombofilia facilitadora de tromboembolismo venoso, na presença de FOP, foi considerado criptogênico.

$O$ aplicativo estatístico utilizado para a análise das p ropriedades estáticas e dinâmicas do DTC foi o Epilnfo 6.04d. Para os testes de hipótese utilizamos o MannWhitney U (StatView, versão 5.0.1). A possibilidade de um erro do tipo I foi afastada adotando-se como nível de significância $p<0,05$. As variáveis contínuas são expressa na forma de média \pm desvio padrão (DP).

\section{RESULTADOS}

Inicialmente foram identificados 129 pacientes. Cinco foram excluídos da amostra: quatro por investigação incompleta e um por apresentar teste de bolhas positivo no DTC, mas não ter cooperado para a realização do ecocardiograma transesofágico, restando 124 pacientes para análise. Apenas quatropacientes da presente série tiveram o diagnóstico de AIT, doravante considerados, para efeito de análise, dentro do grupo com AVCI. Do total de pacientes, houve equilíbrio entre o número daqueles com AVCI não criptogênico e criptogênico, com relação de 1:1,13. Dentre aqueles com etiologia definida, houve distribuição muito semelhante entre os AVCls de origem aterosclerótica (grupo
1 de TOAST), cardioembólico (grupo 2) e com duas ou mais causas definidas (grupo 5), todos com cerca de $10 \%$ do total geral, para cada grupo. Tanto os pacientes classificados como criptogênico, ou não, exibiram proporção indistinguível quanto ao sexo, porém com média de idade superior no grupo não criptogênico $(p=0,017)$. Ao contrário, o tempo médio pós $\mathrm{AVCl}$ foi maior naqueles classificados como criptogênicos ( $p=0,0065)$ (Tabela 2$)$.

Realizaram ecocardiograma transesofágico e DTC 76 pacientes $(61 \%)$, somente ecocardiograma transesofágico $23(19 \%)$ e apenas o DTC 25 pacientes $(20 \%)$. Estes pacientes não realizaram ecocardiograma transesofágico por não cooperarem na realização do exame.

Em 66 (53\%) dos 124 casos, o AVCI foi considerado criptogênico. Dentre esses, as alterações do septo interatrial, notadamente o forame oval patente, foram diagnosticadas em 31 casos (47\%). Nos 58 pacientes classificados como não criptogênico as mesmas alterações foram diagnosticadas em apenas 10 casos (17\%), com uma razão de chance de 4,3 (IC95\% 1,7-10,7) (Figura). Apenas dois casos de comunicação interatrial foram diagnosticados, um no grupo criptogênico e outro no grupo não criptogênico.

Aneurisma do septo interatrial esteve presente em apenas $4 \%$ dos pacientes (5/124), todos eles com forame oval patente. $O$ shunt foi considerado moderado em um dos casos e importante nos outros quatro. Além disso, durante o exame, o shunt sempre esteve presente, independente da manobra

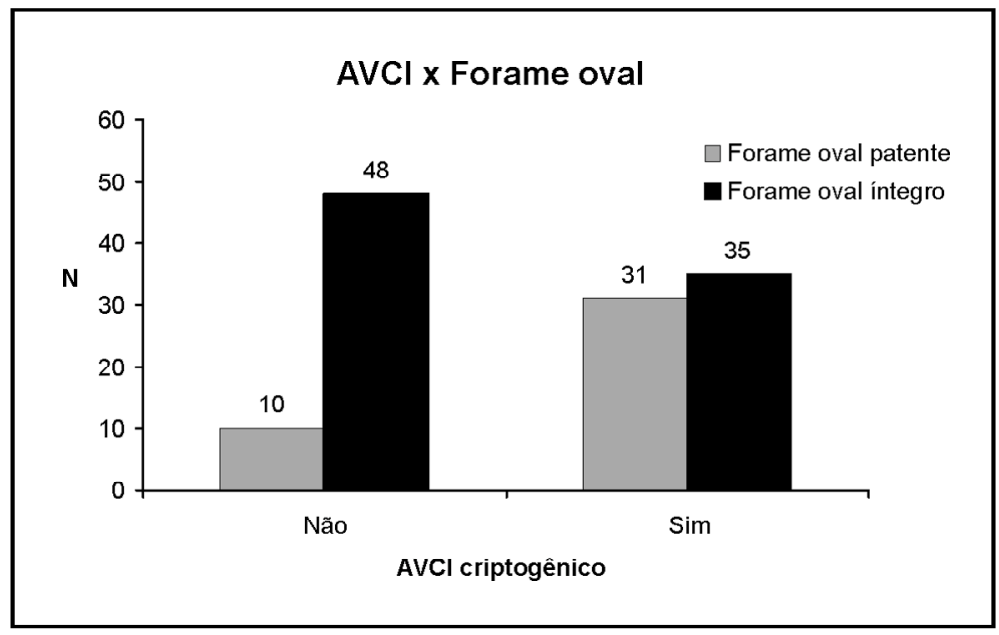

Fig. Freqüência absoluta de pacientes com acidente vascular cerebral isquêmico, criptogênico ou não, em função da presença ou não de shunt direito-esquerdo intracardíaco (forame oval patente ou íntegro). Os números acima das colunas co rrespondem aos valores exatos. Razão de chance $=4,3$ (Intervalo de con fiança 95\% 1,7 - 10,7). 
Tabela 1. Classificação de TOAST ${ }^{12}$ (modificada) de subtipos de AVCI.

1. Aterosclerose de grande artéria

2. Cardioembolismo (excluindo casos atribuídos ao FOP/CIA)

3. Oclusão de pequeno vaso (lacunar)

4. $\quad \mathrm{AVCl}$ de outra etiologia (determinada)

5. $\quad \mathrm{AVCl}$ de etiologia indeterminada. Duas ou mais causas identificadas

6. $\quad \mathrm{AVCl}$ criptogênico

Tabela 2. Características antropométricas, tempo de AVCl e distribuição segundo a classificação TOAST, dicotomizados quanto a definição etiológica (criptogênico ou não).

\begin{tabular}{|c|c|c|c|c|}
\hline Características & $\begin{array}{l}\text { Não criptogênico } \\
\qquad n=58\end{array}$ & & $\begin{array}{l}\text { Criptogênico } \\
\qquad n=66\end{array}$ & $\begin{array}{l}\text { Todos } \\
n=124\end{array}$ \\
\hline Média de idade $( \pm D P)$ & $38,4(9,0)$ & $\dagger$ & $33,6(12,1)$ & $38,0(10,0)$ \\
\hline Sexo feminino (\%) & $30(52)$ & & $39(59)$ & 69 \\
\hline Anos de $\mathrm{AVCl}( \pm \mathrm{DP})$ * & $1,1(1,6)$ & $\dagger$ & $3,0(4,7)$ & $2,1(3,7)$ \\
\hline \multicolumn{5}{|l|}{ TOAST $(\%)$} \\
\hline 1 & 15 & & - & $15(12,1)$ \\
\hline 2 & 15 & & - & $15(12,1)$ \\
\hline 3 & 6 & & - & $6(5,0)$ \\
\hline 4 & 9 & & - & $9(7,3)$ \\
\hline 5 & 13 & & - & $13(10,5)$ \\
\hline 6 & - & & 66 & $66(53,2)$ \\
\hline
\end{tabular}

*Em dois pacientes, em cada grupo, não foi possível precisar a idade por ocasião do AVCl; $\dagger p<0,05$ entre os grupos.

Tabela 3. Validade do doppler transcraniano (DTC) no diagnóstico de shunt direito-esquercbem pacientes com acidente vascular cerebral isquêmico, tendo como padrão-ouro o ecocardiograma transesofágico.

\begin{tabular}{lccc}
\hline \multicolumn{1}{c}{ DTC } & \multicolumn{2}{c}{ Eco transesofágico } & Total \\
& Positivo & Negativo & \\
\multicolumn{1}{c}{ Positivo } & 29 & 5 & 34 \\
$\quad$ Negativo & 1 & 40 & 41 \\
$\quad$ Total & 30 & 45 & 75 \\
\hline Propriedades estáticas e dinâmicas (IC 95\%) & & \\
Sensibilidade & $97 \%$ & $(80,9-99,8)$ \\
Especificidade & $89 \%$ & $(75,2-95,8)$ \\
Valor preditivo positivo & $85 \%$ & $(68,2-94,5)$ \\
Valor preditivo negativo & $98 \%$ & $(85,6-99,9)$ \\
Razão de verossimilhança positiva & 8,7 & $(3,8-19,9)$ \\
Razão de verossimilhança negativa & 0,04 & $(0,005-0,26)$ \\
Concordância & $92 \%$ & $(83,4-97,0)$ \\
Prevalência & $40,0 \%$ & $(28,9-52,0)$ \\
\hline
\end{tabular}

de Valsalva. A média do diâmetro do forame oval na presença de aneurisma do septo interatrial foi $5,2 \mathrm{~mm}( \pm 1,2)$, enquanto que na sua ausência foi $3,6 \mathrm{~mm}( \pm 1,9)(P=0,0623)$. A mensuração do diâmetro do forame oval não foi possível em apenas três dos casos analisados, todos sem aneurisma do septo interatrial.

A Tabela 3 mostra a acurácia do DTC. Tanto a sensibilidade como a especificidade exibiram valores semelhantes e bastante satisfatórios, respec- 
tivamente, $97 \%$ e $89 \%$. O mesmo ocorreu para os valores preditivos positivo e negativo ( $85 \%$ e $98 \%$ ).

\section{DISCUSSÃO}

A presente investigação mostrou, pela primeira vez em nosso meio, uma forte associação entre alterações do septo interatrial, notadamente o FOP, e AVCl em paciente com menos de 51 anos de idade. Paralelamente, os resultados reforçaram a alta validade que o doppler transcraniano exibe, quando comparado ao ecocardiograma transesofágico, ambos associados ao teste de bolhas, na investigação desses pacientes.

Encontram-se documentados na literatura internacional vários estudos sugerindo uma associação entre FOP e $\mathrm{AVCl}$, onde os principais critérios de causalidade seriam atendidos: gradiente doseresposta, consistência entre estudos, especificidade, associação forte e plausibilidade biológica ${ }^{13,14}$. A maioria das pesquisas sobre o tema são de delineamento transversal e caso-controle ${ }^{13} \mathrm{e}$, somente mais recentemente, alguns outros estudos de coorte vem sendo publicados ${ }^{11,15}$, todos indicativos da possível associação entre $\mathrm{AVCl}$ em jovens e $\mathrm{FOP}^{11,15}$. Em que pese todo esse volume de publicações, permanecem ainda algumas dúvidas, no que tange ao diagnóstico e à conduta terapêutica subseqüente, mais especificamente, a prevenção secundária ${ }^{16}$.

Dentro do atual arsenal propedêutico disponível para a investigação de anormalidades do septo interatrial temos, em ordem crescente de complexidade e grau de intervenção, o ecocardiograma transtorácico, ecocardiograma transesofágico e o cateterismo cardíaco. O ecocardiograma transtorácico em adultos, tradicionalmente, não é considerado um bom exame, apresentando baixa validade para o diagnóstico de transtornos do septo interatrial, particularmente FOP, muito possivelmente relacionado com a janela ultra-sônica, que envolve várias estruturas anatômicas subjacentes ${ }^{17}$. $\mathrm{O}$ ecoc a rdiograma transesofágico, desde que associado ao teste de bolhas, é considerado padrão-ouro ${ }^{6}$. O cateterismo cardíaco, por suas características invasivas, não é cogitado no presente contexto. $O$ DTC vem sendo utilizado como importante ferramenta na detecção de shunts direito-esquerdo, dada a sua facilidade de operação, risco e custo comparativamente baixos ${ }^{18}$. No entanto, ainda não se encontra definido, pelo menos em nosso meio, a validade desse método e o seu real papel na seqüência investigativa do paciente com $\mathrm{AVCl}$. Os resultados de validade do DTC que encontramos no presente estudo (sensibilidade de $97 \%$ e especificidade de $89 \%$ ) estão totalmente de acordo com aqueles relatados na literatura ${ }^{19}$. Em publicação recente, consolidando os resultados de diversos outros estudos, a sensibilidade e especificidade desse método foram, respectivamente, superiore $s$ a $70 \%$ e $95 \%{ }^{19}$. Nesse sentido, vale destacar as limitações metodológicas do nosso estudo, principalmente relacionadas com a ausência de mascaramento entre os examinadores, acesso irrestrito às informações no prontuário e o espectro clínico dos pacientes investigados.

Como foi sugerido na presente investigação, o DTC, desde que realizado por pessoas com treinamento adequado, pode ser utilizado como método de triagem para o diagnóstico de shunt direito-esquerdo, particularmente da patência do forame oval. O único caso que configurou um falso negativo (teste de bolhas negativo no DTC mas com p resença de FOP confirmado ao ecocardiograma transesofágico) merece detalhamento. Trata-se de uma paciente do sexo feminino, 33 anos, vítima de $\mathrm{AVCl}$ em território de artéria cerebral média esquerda com 2 anos de evolução. Na exploração diagnóstica foi classificada como AVCl criptogênico. O ecocardiograma transesofágico evidenciou um FOP pequeno $(1,5 \mathrm{~mm})$, associado a um shunt discreto. Uma possível justificativa para a discordância entreos exames pode residir no fato de que a administração do contraste em ambos foi conduzida por acesso no membro superior, minimizando a chance de detecção de pequenos shunts ao DTC ${ }^{20}$. Há evidências de que o acesso por veia femoral, embora pouco prático, torna o exame mais sensível, possivelmente justificado pelo fato de que o fluxo oriundo da veia cava inferior é direcionado para a fossa oval ${ }^{20}$. Pelo exposto, podemos sugerir que um possível algoritmo de decisão com relação a exploração de FOP em pacientes com menos de 51 anos, vítimas de $\mathrm{AVCl}$, poderia passar pela realização de DTC em todos e, no caso de positividade, subseqüente ecocardiograma transesofágico. $\mathrm{Na}$ situação de negatividade ao DTC, porém, em se tratando de paciente classificado como criptogênico, também haveria a indicação de ecocardiograma transesofágico. Em outras palavras, este último exame poderia ser prescindido, nos casos de DTC negativo, em pacientes jovens com causa definida do $\mathrm{AVCl}$. Importante destacar que as limitações do presente estudo (ausência de mascaramento, acesso irrestrito às informações do pacien- 
te, equipes diferentes conduzindo o DTC) apontam para a sua importante validade externa, ou seja, demonstrando a alta efetividade do DTC na detecção de shunts direito-esquerdo. De qualquer forma, vale destacar que o DTC não descrimina shunts intra e extra-cardíacos (por exemplo, pulmonares).

Como todo teste diagnóstico, sua interpretação deve correr à luz dos achados clínicos. Não se deve esquecer que o FOP estava presente em aproximadamente $25 \%$ das necropsias conduzidas em indivíduos sem anormalidades cardiovasculares ${ }^{21}$. Algumas características morfofuncionais que são bem descritas pela ecocardiografia transesofágica podem definir um forame oval como de risco para recorrência de um AVCl: presença deshunt em repouso, shunt considerado importante, associação com aneurisma de septo interatrial, diâmetro do forame oval maior que $4 \mathrm{~mm}$ e valva de Eustachio proeminente ${ }^{22-25}$. Tais constatações revestem-se de especial importância, como veremos adiante, na decisão das condutas subseqüentes.

Ainda dentro do contexto de diagnóstico, no que tange ao aneurisma do septo interatrial, duas considerações se fazem necessárias. A primeira, diz respeito a baixa freqüência dessa condição entre os pacientes da presente série (5 em 124 casos), situada no limite inferior daquela descrita na literatura ${ }^{11}$. Esse fato, provavelmente, decorreu do critério conservador adotado para estabelecer esse diagnóstico. Em segundo lugar, a constatação de que a magnitude do diâmetro do forame oval foi independente da presença de aneurisma do septo interatrial, contrariando observação anterior, que mostrava nítida associação dessa última condição com diâmetros maiores ${ }^{26}$. Considerando o valor limítrofe de significância encontrado $(p=0,0623)$, um erro do tipo II deve ser considerado.

Estabelecido o papel etiológico do FOP, as opções terapêuticas hoje são a antiagregação plaquetária, uso de anticoagulantes orais e o fechamento do forame oval, via percutânea ou cirúrgica. As melhores opções seriam a anticoagulação oral com antagonistas da vitamina $\mathrm{K}$ ou fechamento definitivo do forame oval, de preferência por via percutânea, em função da menor morbidade, quando comparada ao fechamento cirúrgico ${ }^{5,9}$. Os pacientes que mais se beneficiariam dessa intervenção seriam, além daqueles com as características citadas anteriormente, também aqueles com história positiva de eventos isquêmicos prévios.

Outroponto que merece destaque no presente estudo é o fato dos pacientes não terem sido avaliados na fase aguda do AVCl. Isso se deve as características dos hospitais da Rede SARAH, que admite pacientes na fase tardia, com ênfase na sua reabilitação. Assim, devemos ter presente que os pacientes aqui estudados pertencem a uma coorte de "sob reviventes", ou seja, com um viés de seleção, inerente a estudos conduzidos em ambiente hospitalar ${ }^{27}$. Ainda dentro dessa linha, vale destacar que por tratar-se de uma rede de hospitais especializados, referência nacional, os pacientes aqui admitidos apresentam complexidade clínica maior. Cabe aqui também justificar a razão pela qual consideramos, no presente estudo, apenas pacientes com menos de 51 anos. A literatura usualmente considera limites como 45 ou 55 anos $^{22,28,29}$. Adotamos o presente ponto de corte considerando o fato de que na casuística dos hospitais envolvidos a proporção entre pacientes com AVCl criptogênico e não criptogênico era semelhante abaixo dos 51 anos, permitindo assim maior poder estatístico para o estabelecimento da relação causal sob escrutínio. Em que pese essas considerações, os resulta dos alcançados são consistentes com aqueles registrados na literatura que, acreditamos, vem reforçar a importância do tema.

Em síntese, o presente estudo, mediante um delineamento do tipo transversal, de uma população hospitalar, envolvendo pelo menos quatro unidades federadas brasileiras, corrobora os dados da literatura internacional ao estabelecer uma chance quatro vezes maior da presença de FOP entreindivíduos jovens com $\mathrm{AVCl}$, sem causa aparente. Na investigação desses casos o DTC tem um papel fundamental sem, no entanto, prescindir da ecocardiografia transesofágica. Por último, esperamos que essa publicação venha também despertar em nosso meio a atenção dos especialistas, particularmente cardiologistas e neurologistas, para a importância do tema.

\section{REFERÊNCIAS}

1. Ministério da Saúde. Anuário Estatístico de Saúde no Brasil. 2001.

2. Kittner SJ, Sharkness CM, Sloan MA, et al. Infarcts with a cardiac source of embolism in the NINDS Stroke Data Bank: neurologic examination. Neurology 1992;42:299-302.

3. Ferro JM. Cardioembolic stroke: an update. Lancet Neurol 2003;2:177-188.

4. Lechat P, Mas JL, Lascault G, et al. Prevalence of patent foramen ovale in patients with stroke. N Engl J Med 1988;318:1148-1152.

5. Windecker S, Wahl A, Nedeltchev K, et al. Comparison of medical treatment with percutaneous closure of patent foramen ovale in patients with cryptogenic stroke. J Am Coll Cardiol 2004;44:750-758.

6. Jauss M, Kaps M, Keberle M, Haberbosch W, Dorndorf W. A comparison of transesophageal echocardiography and transcranial Doppler sonography with contrast medium for detection of patent foramen ovale. Stroke 1994;25:1265-1267. 
7. Jauss M, Zanette E. Detection of right-to-left shunt with ultrasound contrast agent and transcranial Doppler sonography. Cerebrovasc Dis 2000;10:490-496.

8. Tatani SB, Fukujima MM, Lima JA, et al. Clinical impact of transesophageal echocardiography in patients with stroke without clinical evidence of cardiovascular sources of emboli. Arq Bras Cardiol 2001;76: 453-461.

9. Torreäo J, Costa N, Duarte L, Tadeu E, Brito J, Nery A. Seleçäo de pacientes para fechamento percutâneo dos defeitos do septo interatrial com a prótese de amplattzer através de um escore ecocardiográfico (EEco). Rev Bras Ecocardiogr 2004;17:57-68

10. Lupetin AR, Davis DA, Beckman I, Dash N. Transcranial Doppler sonography: Part 1. Principles, technique, and normal appearances. RadioGraphics 1995;15:179-191.

11. Mas JL, A rquizan C, Lamy C, et al. Recurrent cerebrovascular events associated with patent foramen ovale, atrial septal aneurysm, or both. N Engl J Med 2001;345:1740-1746.

12. Adams HP. Jr, Bendixen BH, Kappelle LJ, et al. Classification of subtype of acute ischemic stroke. Definitions for use in a multicenter clinical trial. TOAST. Trial of Org 10172 in Acute Stroke Treatment. Stroke 1993; 24:35-41.

13. Overell JR, Bone I, Lees KR. Interatrial septal abnormalities and stroke: a meta-analysis of case-control studies. Neurology 2000;55:1172-1179.

14. Grimes DA, Schulz KF. Bias and causal associations in observational research. Lancet 2002;359:248-252.

15. Windecker S, Wahl A, Chatterjee T, et al. Percutaneous closure of patent foramen ovale in patients with paradoxical embolism: long-term risk of recurrent thromboembolic events. Circulation 2000;101:893-898.

16. Castello R, Brott TG. Patent foramen ovale: friend or foe? J Am Coll Cardiol 2003;42:1073-1075.

17. Belkin RN, Pollack BD, Ruggiero ML, Alas LL, Tatini U. Comparison of transesophageal and transthoracic echocardiography with contrast and color flow Doppler in the detection of patent foramen ovale. Am Heart J 1994;128:520-525.

18. D roste DW, Schmidt-Rimpler C, Wichter T, et al. Right-to-left-shunts detected by transesophageal echocardiography and transcranial Doppler sonography. Cerebrovasc Dis 2004;17:191-196.
19. Sloan MA, Alexandrov AV, Tegeler $\mathrm{CH}$, et al. Assessment: transcranial Doppler ultrasonography: report of the Therapeutics and Technology Assessment Subcommittee of the American Academy of Neurology. Neurology 2004;62:1468-1481.

20. Hamann GF, Schatzer-Klotz D, Frohlig G, et al. Femoral injection of echo contrast medium may increase the sensitivity of testing for a patent foramen ovale. Neurology 1998;50:1423-1428.

21. Hagen PT, Scholz DG, Edwards WD. Incidence and size of patent foramen ovale during the first 10 decades of life: an autopsy study of 965 normal hearts. Mayo Clin Proc 1984;59:17-20.

22. Mesa D, Franco M, Suarez DL, et al. Prevalence of patent foramen ovale in young patients with cerebral ischemic accident of unknown origin. Rev Esp Cardiol 2003;56:662-668.

23. Schuchlenz HW, Weihs W, Horner S, Quehenberger F. The association between the diameter of a patent foramen ovale and the risk of embolic cerebrovascular events. Am J Med 2000;109:456-462.

24. Steiner MM, Di Tullio MR, Rundek T, et al. Patent foramen ovale size and embolic brain imaging findings among patients with ischemic stroke. Stroke 1998;29:944-948.

25. Schuchlenz HW, Saurer G, Weihs W, Rehak P. Persisting eustachian valve in adults: relation to patent foramen ovale and cerebrovascular events. J Am Soc Echocardiogr 2004;17:231-233.

26. Fox ER, Picard MH, Chow CM, Levine RA, Schwamm L, Kerr AJ. Interatrial septal mobility predicts larger shunts across patent foramen ovales: an analysis with transmitral Doppler scanning. Am Heart J 2003;145:730-736.

27. PMID: 7214 286, PubMed. How to read clinical journals: III. To learn the clinical course and prognosis of disease. Can Med Assoc J 1981;124:869-872.

28. Cerrato P, Grasso M, Imperiale D, et al. Stroke in young patients: etiopathogenesis and risk factors in diffe rent age classes. Cere brovasc Dis 2004;18:154-159.

29. Mattioli AV, Bonetti L, Aquilina M, Oldani A, Longhini C, Mattioli G. Association between atrial septal aneurysm and patent foramen ovale in young patients with recent stroke and normal carotid arteries. Cerebrovasc Dis 2003;15:4-10. 